\title{
A general pattern of the species abundance distribution
}

\author{
Qiang Su Corresp. 1 \\ ${ }^{1}$ College of Earth and Planetary Sciences, University of Chinese Academy of Sciences, Beijing, China \\ Corresponding Author: Qiang Su \\ Email address: sqiang@ucas.ac.cn
}

Since the 1970s, species abundance distributions (SADs) have been one of the most fundamental issues in ecology and have frequently been investigated and reviewed. However, there was surprisingly little consensus. This study focuses on three essential questions. (1) Is there a general pattern of SAD that no community can violate it? (2) If it exists, what does it look like? (3) Why is it like this? The frequency distributions of 19,833 SADs from eight datasets (including eleven taxonomic groups from terrestrial, aquatic, and marine ecosystems) suggest that a general pattern of SAD might exist. According to two hypotheses (the finiteness of the total energy and the causality from the entropy to the diversity), this study assumes that the general pattern of SAD is approximately consistent with Zipf's law, which means that Zipf's law might be more easily to observe when one investigates any SAD. In the further, this conjecture not only needs to be tested (or supported) by more and more datasets, but also depends on how well it is explained from different angles of theories. 


\section{A general pattern of the species abundance 2 distribution}

3 Qiang $\mathrm{Su}^{*}$, a

4 a, College of Earth and Planetary Sciences (CEPS), University of Chinese

5 Academy of Sciences (UCAS), No.19A Yuquan Road, Beijing, 100049, China

6 sqiang@ucas.ac.cn

$7 \quad+861088256997$

8

9 Running Head: Diversity and Zipf's law.

10

11 Corresponding author: Dr. Qiang Su

12 Words in the Abstract: 176

13 Words in main body: 3114

14 References: 29

15 Table: 2

16 Figures: 1 


\section{Abstract}

Since the 1970s, species abundance distributions (SADs) have been one of the

20 most fundamental issues in ecology and have frequently been investigated and

21 reviewed. However, there was surprisingly little consensus. This study focuses on

22 three essential questions. (1) Is there a general pattern of SAD that no community

23 can violate it? (2) If it exists, what does it look like? (3) Why is it like this? The

24 frequency distributions of 19,833 SADs from eight datasets (including eleven

25 taxonomic groups from terrestrial, aquatic, and marine ecosystems) suggest that a

26 general pattern of SAD might exist. According to two hypotheses (the finiteness of

27 the total energy and the causality from the entropy to the diversity), this study

28 assumes that the general pattern of SAD is approximately consistent with Zipf's

29 law, which means that Zipf's law might be more easily to observe when one

30 investigates any SAD. In the further, this conjecture not only needs to be tested (or

31 supported) by more and more datasets, but also depends on how well it is

32 explained from different angles of theories.

33 


\section{INTRODUCTION}

37 Species abundance distributions (SADs) describe the distribution of

38 commonness and rarity in a community (McGill et al. 2007, Baldridge et al. 2016).

39 McGill et al. (2007) stated that "understanding SAD is a major stepping stone to

40 understanding communities in general". SADs play a central role in ecology

41 because the determinants of diversity also depend on how well SADs are explained

42 (May 1975, Magurran 1988, Tokeshi 1993, McGill et al. 2007, Baldridge et al.

43 2016).

44 Since the 1970s, numerous SAD models have been proposed on various

45 theoretical grounds, and they were observed in real situations (May 1975, Frontier

46 1987, Magurran 1988, Tokeshi 1993, Mouillot et al. 2000, McGill et al. 2007,

47 Baldridge et al. 2016, Su 2016). However, it is difficult to draw general

48 conclusions about which models provide the best fit to SADs (Baldridge et al.

49 2016). Although many ecologists hoped that distinguishing subtle variations in

50 these models would provide a decisive test, this had not worked well (McGill et al.

51 2007).

52 Baldridge et al. (2016) pointed out that the log-series model provided a slightly

53 better fit to the abundance distributions of over 16,000 community samples.

54 Connolly et al. (2014) suggested the Poisson lognormal model as an appropriate

55 description of 1,185 SADs from 14 marine ecosystems. A similar study according 
56 to 558 samples indicated that lognormal type SADs fitted much better than log-

57 series model or the Zipf model (Ulrich et al. 2010). In brief, SAD models predicted

58 very similar shapes and their distinction became problematic (May 1975, Magurran

59 1988, Tokeshi 1993, McGill et al. 2007, Ulrich et al. 2010, Connolly et al. 2014,

60 Baldridge et al. 2016).

61 Baldridge et al. (2016) suggested that the SAD usually did not contain

62 sufficient information to distinguish among different models and a more promising

63 way was to evaluate each model's ability to simultaneously explain multiple

64 macroecological patterns. In fact, when one investigates any community, it is

65 ubiquitously observed that many species are rare and just a few are common (May

66 1975, Frontier 1987, Magurran 1988, Tokeshi 1993, Mouillot et al. 2000, McGill

67 et al. 2007, Baldridge et al. 2016, Su 2016). Although there is a great variability in

68 the observed SAD, such consistent pattern appears to be very universal (May 1975,

69 Magurran 1988, Tokeshi 1993, McGill et al. 2007, Ulrich et al. 2010, Connolly et

70 al. 2014, Baldridge et al. 2016).

71 The purpose of this study is no longer the comparison of SAD models. The

72 main questions are that (1) is there (or is there not) a general pattern of SAD that

73 no community (from the marine benthos to the Amazonian rainforest) can violate it?

74 (2) if such pattern exists, what does it look like? (3) why is a community generally

75 organized in such pattern? To this end, the frequency distributions of 19,833 SADs 
76 from eight datasets (including eleven taxonomic groups from terrestrial, aquatic,

77 and marine ecosystems) are evaluated.

78 MeTHODS

79 Since SAD models all can describe the pattern that many species are rare and

80 just a few are common, a new fractal model of SAD ( $\mathrm{Su} 2016)$ is selected for three

81 reasons. (1) Its hypothesis is easy to fit (Frontier 1987, Mouillot et al. 2000); (2)

82 The frequency of SADs is easy to obtain as there is only one parameter in this

83 model (Su 2016); (3) The extension of this model into a larger ecological context

84 and its ability to explain multiple ecological patterns have not been fully

85 understood.

86 The theoretical bases of this model have been given by Frontier (Frontier 1985,

87 Frontier 1987, 1994). In ecology, its interpretation leaded to two different but non-

88 contradictory interpretations (Zipf 1949, Mandelbrot 1953, Frontier 1987, 1994,

89 Mouillot et al. 2000, Su 2016). One was the "cost of a species", which was linked

90 with the amount of assimilated energy that it required. For example, it is costlier in

91 terms of energy for an ecosystem to produce and maintain a carnivore than a

92 primary producer, because of the loss of energy at each trophic level (Frontier

$931987,1994)$. The other referred to the existence of previous conditions allowing

94 the presence of a species (Frontier 1987, 1994, Su 2016). For example, some of

95 these conditions may be the occurrences of some previous species in the ecological 
96 succession since any species modifies the biological and physical environment,

97 permitting or hindering another species to appear (Frontier 1985, Frontier 1987,

98 1994).

99 According to the original hypothesis (when $K$ more species appear at each step 100 of the accumulation process, their abundance are $k$ times less abundant and $K=k^{d}$,

101 where $d(>0)$ is a fractal dimension (Mouillot et al. 2000)), SAD in a community is

$102 \quad \frac{A_{r}}{A_{1}}=r^{-p}$

103 where $r(=1,2,3, \ldots S)$ is the rank of species sorted down by species abundance; $A_{1}$

104 and $A_{r}$ are the abundance of dominant and the $r$-th species; $p(=1 / d)$ is the fractal

105 parameter, which determines the pattern of the SAD ( $\mathrm{Su} 2016)$. For example, when

$106 p=1$ and $S=6, \operatorname{SAD}\left(A_{r} / A_{1}\right)$ is

$107 \quad 1,1 / 2,1 / 3,1 / 4,1 / 5,1 / 6$

108 Let $F_{r}=\ln \left(A_{r} / A_{1}\right)$ and $D_{r}=\ln (r)$. By minimizing the sum of squared errors (

$\left.109 \sum_{r=1}^{S}\left(-p D_{r}-F_{r}\right)^{2}\right), p$ is estimated as follows

$110 p=\frac{-\sum_{r=1}^{S} D_{r} F_{r}}{\sum_{r}^{S} D_{r}^{2}}$

111 Similarly, if $\operatorname{SAD}\left(A_{r} / A_{1}\right)$ in a community is $1,1 / 2,1 / 3,1 / 4,1 / 5,1 / 6$, the fractal

$112 p$ is 1 according to $E q$. (2).

113 The sum of Eq. (1) is

$114 \quad \frac{A_{T}}{A_{1}}=\sum_{r=1}^{S} r^{-p}$ 
115 where $A_{T}$ is the total abundance.

116 According to Hill's notation that is related to Rényi's definition of a

117 generalized entropy, $A_{T} / A_{1}$ is an effective number of species with the order $a=\infty$

118 (Rényi 1961, Hill 1973). When $p=1$, the difference between $A_{T} / A_{1}$ and $\ln (S)$ in

119 mathematic converges to the Euler-Mascheroni constant.

120 If $S$ is infinite, $E q .(3)$ is

$121 \quad \frac{A_{T}}{A_{1}}=\sum_{r=1}^{\infty} r^{-p}$

$122 E q$. (4) is consistent with the generalization of the harmonic series. It converges

123 for all $p>1$ and diverges for $p \leq 1$. When $p=1, E q$. (4) is the observed Zipf's law (or

124 Zipf distribution) (Zipf 1949, Seuront 2009).

125 In brief, Eq. (1) is based on the fractal hypothesis of diversity (Frontier 1987)

126 to create a mathematic link between the Rényi’s entropy (Rényi 1961, Hill 1973)

127 and an empirical distribution (Zipf's law) (Zipf 1949).

128 Datasets

129 Eight datasets (named "fish", "diatom", "nabc", "mcdb", "gentry", "fia", "cbc"

130 and "bbs") from two sources (Baldridge et al. 2016, Passy 2016a) were used for

131 four reasons. (1) These datasets are under different environments with broad

132 representations; (2) They are relatively reliable as they have been used in SAD

133 studies (Baldridge et al. 2016, Passy 2016a); (3) The frequency distributions of $p$ 
134 for these datasets are unclear; (4) Published datasets are easy to recheck. The detail

135 information of these datasets can be found in appendixes of Passy (2016a, b) and

136 Baldridge et al. (2016, 2016, November 15). Briefly, 19,833 quantitative samples

137 from eleven taxonomic groups (representing over 3 billion individual terrestrials,

138 aquatic, and marine organisms) were collected to explore the frequency of

139 empirical SADs.

140 Results

141 According to Eq. (2), the fractal $p$ for the entire dataset (Baldridge et al. 2016,

142 Passy 2016a, b, Baldridge et al. 2016, November 15) is from 0.235 to 5.825 (Table

143 1). The quality of fits is measured by $\mathrm{R}^{2}$ (Table 2 ), which denotes the goodness of

144 fit on the $\log$-transformed variables $\left(\log r\right.$ and $\log A_{r} / A_{1}$, please see Code 1 in

145 Supplemental Files). The average and median value of $p$ are $1.108 \pm 0.003$ and

1461.034 , respectively. The highest value of the mean $p(1.702 \pm 0.019)$ is in the "fish"

147 group (Passy 2016b, a). The lowest one is in "fia" group (0.931 \pm 0.003$)$ (Baldridge

148 et al. 2016, 2016, November 15). The median $p$ is from 0.827 to 1.592 . In short,

149 although the range of the fractal $p$ for the entire dataset is over one order of

150 magnitude, the average and median value of $p$ for eight groups are similar (close to

151 1) (Table 1).

152 The frequency distributions of the fractal $p$ for eight groups are presented in 
153 Figure 1. The "diatom" and "nabc" groups show similar frequency distributions,

154 noting that the fractal $p$ centrally occurs in the range from 1 to 1.33 (Figure $1 \mathrm{~A}$

155 and H); For the "bbs", "fia" and "gentry" groups, $p$ is near to 1 (from 0.67 to 1 ,

156 Figure $1 \mathrm{C}, \mathrm{E}$ and F); The frequency distributions of the fractal $p$ for the "fish",

157 "cbc" and "mcdb" groups skew to the higher value that $p$ is from 1.33 to 1.67

158 (Figure $1 \mathrm{~B}, \mathrm{D}$ and $\mathrm{G})$. The consensus of the $p$ distributions for every group is clear

159 that $p$ mostly appears close to 1 . It is rare that the fractal $p$ is far greater than 1 or

160 very near 0 (Figure 1).

161 Briefly, the patterns of $p$ frequencies (Figure 1) and the average and median

162 value of $p$ (Table 1) are consistent, which both suggest that $p$ closer to 1 does seem

163 to be happening more frequently in real situations. The mechanisms underlying the

164 frequency distributions of $p$ might warrant further investigations.

\section{Discussion}

166 "We are all blind men (and women) trying to describe a monstrous elephant of

167 ecological and evolutionary diversity" (Nanney 2004, Chao et al. 2010). No matter

168 how diversity is defined, there is unimaginable variation in the diversity of entire

169 living systems (Pielou 1975, Huston 1994). The general consensus is that an

170 informative way to summarize the characteristics of diversity is not a statistic

171 index (e.g. Shannon's index or Simpson's index) but the species abundance

172 distributions (SADs) (Pielou 1975, Frontier 1987, Magurran 1988, Tokeshi 1993). 
173 Countless investigations and comparisons of the SAD models have been explored

174 from different angles of theories (May 1975, Frontier 1987, Magurran 1988,

175 Tokeshi 1993, Mouillot et al. 2000, McGill et al. 2007, Baldridge et al. 2016, Su

176 2016). Unfortunately, it hardly worked (McGill et al. 2007, Baldridge et al. 2016).

177 Thus, as noted before, the main purposes of this study shift to three fundamental

178 questions. (1) Is there a consistent general pattern of SADs? (2) If it exists, what

179 does it look like? (3) Why is it like this?

180 Firstly, according to following three reasons, this study suggests that a general

181 pattern of SADs might exist. (1) The number of community samples in this study is

182 relatively adequate. Nearly 20,000 quantitative samples are used to explore the

183 frequency distributions of SADs. (2) The sources of datasets are extensive,

184 including terrestrial, aquatic, and marine ecosystems (Baldridge et al. 2016, Passy

185 2016a, b, Baldridge et al. 2016, November 15). The above two points indicate that

186 the datasets used in this paper have broad representation and the frequency

187 distributions of SADs based on such datasets are reliable. (3) If the general pattern

188 of SADs does not exist, the frequency distributions of the fractal $p$ will be discrete

189 and irregular, or it will be quite different for eight groups. However, $p$ distributions

190 for different taxonomic categories and groups show a consistent pattern. It is very

191 rare that the fractal $p$ is far greater than 1 or very near 0 , and $p$ closer to 1 is the

192 most common case (Figure 1). 
193 Secondly, it might be very hard to draw a definite conclusion about which

194 pattern is the general SAD. On one hand, for the entire dataset, the average and

195 median value of $p(1.108 \pm 0.003$ and 1.034$)$ are both slightly higher than 1 (Table

196 1). The fractal $p$ of every group occurs frequently in a similar range (close to 1)

197 (Figure 1). On the other hand, the peaks of $p$ distributions for eight groups are not

198 exactly the same. It could be from 0.67 to 1 (Figure $1 \mathrm{C}, \mathrm{E}$ and F), or from 1.33 to

1991.67 (Figure 1 B, D and G). Accordingly, this study assumes that the general

200 pattern of SAD is that the fractal $p$ exceeds and approaches 1 . This conjecture not

201 only needs to be supported by further investigations and additional datasets, but

202 also depends on how well it is explained in theory.

203 Finally, if the general pattern is that $p$ exceeds and approaches 1 , how is it

204 understood by current theories? In fact, such distribution $(p \approx 1$, see Eq. (1)) is

205 approximately consistent with Zipf's law (or Zipf distribution) (Zipf 1949,

206 Mandelbrot 1953, Frontier 1987, Seuront 2009). In communication systems, Zipf's

207 law holds for almost all languages' letters and words (Seuront 2009). Zipf stated

208 that this empirical distribution attributed to the "Principle of Least Effort",

209 representing a balance between the repetition desired by the listener and the

210 diversity desired by the transmitter (Zipf 1949, Seuront 2009). If a repertoire is too

211 repetitive, a communication is sent by a few signals, and less message is conveyed.

212 Alternatively, if a repertoire is too diverse, the same message can be 
213 overrepresented by a multitude of signals, and less communication is conveyed.

214 These opposite forces result in a balance between unification and diversification

215 (Zipf 1949, Seuront 2009).

216 However, this principle seems hard to explain the general pattern of SAD from

217 the perspective of ecology (Mandelbrot 1953, Frontier 1987). Later, Zipf's law was

218 modified by Mandelbrot as $f_{r}=f_{0}(r+\beta)^{-\alpha}$ (Mandelbrot 1953, Frontier 1987,

219 Seuront 2009). In ecology, $f_{r}$ was the frequency of the $r$-th species after ranking the

220 species in decreasing order. $f_{0}$ is chosen such that the sum of all $f_{r}$, values predicted

221 by the model is 1 (Frontier 1987). The $\alpha$ and $\beta$ are conditioning the species

222 diversity and the evenness of a given community (Frontier 1987, Seuront 2009).

223 Unfortunately, the Mandelbrot model might be unable to fully explain the

224 underlying mechanisms of the general SAD (Mandelbrot 1953, Frontier 1987,

225 Seuront 2009, Su 2016).

226 This study proposes two hypotheses to elucidate the general pattern of SAD ( $p$

227 exceeds and approaches 1). (1) The total abundance ( $A_{T}$, see $E q$. (4)) is equivalent

228 to the total assimilated energy of the community, which is finite for a given

229 condition. (2) The abundance of each species is linked with the energy

230 transformation from the total energy, which increases the community entropy that

231 determines the diversity. On one hand, the theoretical bases of two hypotheses

232 actually derive from the first interpretation of the fractal model (see the second 
233 paragraph of Methods), noting that a species is linked with the amount of

234 assimilated energy (Zipf 1949, Mandelbrot 1953, Frontier 1987, 1994, Mouillot et

235 al. 2000, Su 2016). The noticeable differences between the first interpretation and

236 two hypotheses are the finiteness of the total energy and the causality from the

237 community entropy to the diversity. On the other hand, quantifying diversity

238 according to the entropy (e.g. Shannon's entropy and Rényi's entropy) is not new

239 (Rényi 1961, Hill 1973, Magurran 1988, Tuomisto 2012). Therefore, two

240 hypotheses are not contradictory with current fractal and diversity theories

241 (Mandelbrot 1953, Rényi 1961, Hill 1973, Frontier 1987, Magurran 1988).

242 According to the first hypothesis, $A_{T}$ is finite. Thus, $A_{T} / A_{1}$ is also finite as $A_{1}$ is

243 the abundance of dominant (see $E q$. (1)). The finiteness of $A_{T} / A_{1}$ determines that

244 the fractal $p$ should be higher than 1 because $A_{T} / A_{1}$ converges for all $p>1$ (see $E q$.

245 (4)). According to the second hypothesis, the diversity generally presents a trend of

246 increasing because the energy conversion among species increases the community

247 entropy. If the community entropy can be expressed as Hill's unifying notation and

248 Rényi’s entropy (Rényi 1961, Hill 1973, Jost 2006, Chao et al. 2010, Jost 2010,

249 Chao et al. 2014, Chao and Jost 2015), $A_{T} / A_{1}$ tends to increase with the entropy and

250 diversity because $A_{T} / A_{1}$ is an effective number of species (Rényi 1961, Hill 1973).

251 An increasing trend of $A_{T} / A_{1}$ means a decreasing trend of $p$ because the fractal $p$ is

252 negative with $A_{T} / A_{1}$ (see $E q$. (4)). Therefore, the balance between two forces (the 
253 fractal $p$ is higher than 1 and tends to decline) eventually leads to the general 254 pattern of SADs that $p$ exceeds and approaches 1.

255 


\section{CONCLUSIONS}

257 When one investigates any SAD, previous studies suggested that it was

258 ubiquitously observed that many species were rare and just a few were common

259 (May 1975, Frontier 1987, Magurran 1988, Tokeshi 1993, Mouillot et al. 2000,

260 McGill et al. 2007, Baldridge et al. 2016, Su 2016). However, this study indicates

261 that it is more easily to observe that the fractal $p(\mathrm{Su} 2016)$ exceeds and approaches

262 1. This is the biggest difference between previous studies and this paper.

263 It might be a surprise that the fractal model has been around for some time but

264 it is not widely used by ecologists (Zipf 1949, Mandelbrot 1953, Frontier 1987,

265 1994, Mouillot et al. 2000, Su 2016). There were five families with over 40 SAD

266 models, and it might be normal that some of them were not well known (McGill et

267 al. 2007). Tokeshi (1993) commented that the fractal model was no more

268 biological than others. However, such views were lack of empirical investigations

269 (Tokeshi 1993). In fact, there was a good fit of the fractal SAD to raw data

270 (Frontier 1987, 1994, Mouillot et al. 2000, Seuront 2009, Su 2016), and its

271 theoretical bases had been elaborated (Frontier 1985, Frontier 1987, 1994, Mouillot

272 et al. 2000). In the future, a more promising way is likely to evaluate the

273 performance of the fractal model and increase the understanding of mechanisms

274 that lead to the general pattern of SAD. 


\section{ACKNOWLEDGMENTS}

277 My deepest gratitude goes to Marcio Pie (editor), Juan Pablo Gomez (reviewer)

278 and an anonymous reviewer for their careful work and thoughtful suggestions that

279 have helped improve this paper substantially.

280 


\section{REFERENCES}

282 1. Baldridge, E., D. J. Harris, X. Xiao, and E. P. White. 2016. An extensive 283 comparison of species-abundance distribution models. Peerj 4.

284 2. Baldridge, E., D. J. Harris, X. Xiao, and E. P. White. 2016, November 15. 285 weecology/sad-comparison: First revision for PeerJ (Version peerj.2). Zenodo.

286 3. Chao, A., C.-H. Chiu, and L. Jost. 2010. Phylogenetic diversity measures 287 based on Hill numbers. Philosophical Transactions of the Royal Society of 288 London B: Biological Sciences 365:3599-3609.

289 4. Chao, A., and L. Jost. 2015. Estimating diversity and entropy profiles via 290 discovery rates of new species. Methods in Ecology and Evolution 6:873-882.

291 5. Chao, A. N., C. H. Chiu, and L. Jost. 2014. Unifying Species Diversity, 292 Phylogenetic Diversity, Functional Diversity, and Related Similarity and 293 Differentiation Measures Through Hill Numbers. Pages 297-324 in D. J. 294 Futuyma, editor. Annual Review of Ecology, Evolution, and Systematics, Vol 295 45. Annual Reviews, Palo Alto.

296 6. Connolly, S. R., M. A. MacNeil, J. Caley, N. Knowlton, E. Cripps, M. Hisano, 297 L. M. Thibaut, B. D. Bhattacharya, L. Benedetti-Cecchi, R. E. Brainard, A.

298 Brandt, F. Bulleri, K. E. Ellingsen, S. Kaiser, I. Kroncke, K. Linse, E. Maggi, 299 T. D. O'Hara, L. Plaisance, G. C. B. Poore, S. K. Sarkar, K. K. Satpathy, U. 300 Schuckel, A. Williams, and R. S. Wilson. 2014. Commonness and rarity in 
301 the marine biosphere. Proceedings of the National Academy of Sciences of

302 the United States of America 111:8524-8529.

303 7. Frontier, S. 1985. Diversity and structure in aquatic ecosystems.

304 Oceanography and Marine Biology 23:253-312.

305 8. Frontier, S. 1987. Applications of fractal theory to ecology. Pages 335-378

306 Develoments in Numerical Ecology. Springer.

307 9. Frontier, S. 1994. Species diversity as a fractal property of biomass. Pages

308 119-127 in In: Fractals in the Natural and Applied Sciences (ed. Novak, M.).

$309 \quad$ North-Holland Publishing, Amsterdam.

310 10. Hill, M. O. 1973. Diversity and evenness - unifying notation and its

$311 \quad$ consequences. Ecology 54:427-432.

312 11. Huston, M. A. 1994. Biological diversity: the coexistence of species on

313 changing landscapes. Cambridge University Press.

314 12. Jost, L. 2006. Entropy and diversity. Oikos 113:363-375.

315 13. Jost, L. 2010. The relation between evenness and diversity. Diversity 2:207-

316232.

317 14. Magurran, A. E. 1988. Ecological Diversity and Its Measurement. Princeton

$318 \quad$ University Pre, New Jersey.

319 15. Mandelbrot, B. 1953. An informational theory of the statistical structure of

$320 \quad$ language. Communication theory 84. 
321 16. May, R. M. 1975. Patterns of species abundance and diversity. Belknap Press

322 of Harvard University Press, Cambridge MA.

323 17. McGill, B. J., R. S. Etienne, J. S. Gray, D. Alonso, M. J. Anderson, H. K.

324 Benecha, M. Dornelas, B. J. Enquist, J. L. Green, F. He, A. H. Hurlbert, A. E.

325 Magurran, P. A. Marquet, B. A. Maurer, A. Ostling, C. U. Soykan, K. I.

326 Ugland, and E. P. White. 2007. Species abundance distributions: moving

327 beyond single prediction theories to integration within an ecological

328 framework. Ecology Letters 10:995-1015.

329 18. Mouillot, D., A. Lepretre, M. C. Andrei-Ruiz, and D. Viale. 2000. The Fractal

330 Model: a new model to describe the species accumulation process and relative

331 abundance distribution (RAD). Oikos 90:333-342.

332 19. Nanney, D. L. 2004. No trivial pursuit. Bioscience 54:720-721.

333 20. Passy, S. I. 2016a. Abundance Inequality in Freshwater Communities Has an

334 Ecological Origin. The American Naturalist 187:502-516.

335 21. Passy, S. I. 2016b. Data from: Abundance inequality in freshwater

336 communities has an ecological origin. Dryad Data Repository.

337 22. Pielou, E. 1975. Ecological diversity.

338 23. Rényi, A. 1961. On measures of entropy and information. Pages 547-561 in

339 Fourth Berkeley symposium on mathematical statistics and probability.

340 24. Seuront, L. 2009. Fractals and multifractals in ecology and aquatic science. 
$341 \quad$ CRC Press.

342 25. Su, Q. 2016. Analyzing fractal property of species abundance distribution and

343 diversity indexes. Journal of Theoretical Biology 392:107-112.

344 26. Tokeshi, M. 1993. Species abundance patterns and community structure.

345 Advances in ecological research 24:111-186.

346 27. Tuomisto, H. 2012. An updated consumer's guide to evenness and related 347 indices. Oikos 121:1203-1218.

348 28. Ulrich, W., M. Ollik, and K. I. Ugland. 2010. A meta-analysis of species-

349 abundance distributions. Oikos 119:1149-1155.

350 29. Zipf, G. K. 1949. Human behavior and the principle of least effort. 


\section{Table $\mathbf{1}$ (on next page)}

The detailed information of the fractal $p$ (Su 2016) for eight datasets (named "fish", "diatom", "nabc", "mcdb", "gentry", "fia", "cbc" and "bbs") from two sources (Baldridge et al. 2016a, b, Passy 2016a, b).

The average and median value of $p$ for the entire dataset are $1.108 \pm 0.003$ and 1.034, respectively. Although the range of the fractal $p$ is over one order of magnitude (from 0.235 to 5.825), the average and median value of $p$ for eight groups are consistent, noting that they are close to 1 . 


\section{TABLe Legends}

2 Table 1 The detailed information of the fractal $p(\mathrm{Su} 2016)$ for eight datasets

3 (named "fish", "diatom", "nabc", "mcdb", "gentry", "fia", "cbc" and "bbs") from

4 two sources (Baldridge et al. 2016a, b, Passy 2016a, b). The average and median

5 value of $p$ for the entire dataset are $1.108 \pm 0.003$ and 1.034, respectively. Although

6 the range of the fractal $p$ is over one order of magnitude (from 0.235 to 5.825), the

7 average and median value of $p$ for eight groups are consistent, noting that they are

8 close to 1 .

9 
10 Table 1

\begin{tabular}{lccccc}
\hline Fractal $p$ & Maximum & Minimum & Median & Average & Sample numbers \\
\hline diatom & 5.825 & 0.335 & 1.272 & $1.343 \pm 0.008$ & 3224 \\
fish & 4.563 & 0.756 & 1.592 & $1.702 \pm 0.019$ & 761 \\
bbs & 2.375 & 0.548 & 0.938 & $0.984 \pm 0.004$ & 2769 \\
cbc & 3.738 & 0.733 & 1.492 & $1.556 \pm 0.008$ & 1999 \\
fia & 2.229 & 0.235 & 0.907 & $0.931 \pm 0.003$ & 10355 \\
gentry & 1.851 & 0.352 & 0.827 & $0.872 \pm 0.019$ & 222 \\
mcdb & 3.265 & 0.495 & 1.547 & $1.587 \pm 0.052$ & 103 \\
nabc & 3.112 & 0.540 & 1.240 & $1.278 \pm 0.017$ & 400 \\
Total & 5.825 & 0.235 & 1.034 & $1.108 \pm 0.003$ & 19833 \\
\hline
\end{tabular}




\section{Table 2 (on next page)}

The goodness of fit $\left(R^{2}\right)$ of the fractal model

The goodness of fit ( $R^{2}$, please see Code 1 in Supplemental Files) of the fractal model (Su 2016) on each of 19,833 samples are shown in the following table (the range in the "Average" column is standard error). $\mathrm{R}^{2}$ varies between 0 and 1 , with larger numbers indicating better fits. 


\section{TABle Legends}

2 Table 2 The goodness of fit $\left(\mathrm{R}^{2}\right.$, please see Code 1 in Supplemental Files) of the

3 fractal model ( $\mathrm{Su} 2016)$ on each of 19,833 samples are shown in the following

4 table (the range in the "Average" column is standard error). $\mathrm{R}^{2}$ varies between 0

5 and 1, with larger numbers indicating better fits.

6 
$7 \quad$ Table 2

\begin{tabular}{cccccc}
\hline $\mathrm{R}^{2}$ & Maximum & Minimum & Median & Average & \multicolumn{2}{c}{ Sample numbers } \\
\hline diatom & 1.000 & 0.460 & 0.917 & $0.901 \pm 0.001$ & 3224 \\
fish & 0.997 & 0.397 & 0.841 & $0.829 \pm 0.004$ & 761 \\
bbs & 0.982 & 0.555 & 0.792 & $0.791 \pm 0.001$ & 2769 \\
cbc & 0.990 & 0.474 & 0.787 & $0.785 \pm 0.002$ & 1999 \\
fia & 0.989 & 0.089 & 0.880 & $0.860 \pm 0.001$ & 10355 \\
gentry & 0.969 & 0.640 & 0.911 & $0.892 \pm 0.004$ & 222 \\
mcdb & 0.981 & 0.466 & 0.869 & $0.838 \pm 0.011$ & 103 \\
nabc & 0.986 & 0.639 & 0.884 & $0.872 \pm 0.004$ & 400 \\
Total & 1.000 & 0.089 & 0.867 & $0.849 \pm 0.001$ & 19833 \\
\hline
\end{tabular}


Figure 1 (on next page)

The frequency distributions of the fractal $p$ (Su 2016) for eight datasets (named "fish", "diatom", "nabc", "mcdb", "gentry", "fia", "cbc" and "bbs") from two sources (Baldridge et al. 2016a, b, Passy 2016a, b).

The peaks of $p$ frequencies for eight groups are not exactly the same. It could be from 0.67 to 1 ( $C, E$ and F), from 1 to 1.33 ( $A$ and $H$ ) and from 1.33 to 1.67 (B, D and $G$ ). However, the consensus of the $p$ frequencies for every group is very clear. The fractal $p$ mostly appears close to 1 , and it is rare that $p$ is far greater than 1 or very near 0 . 
A PeerJ

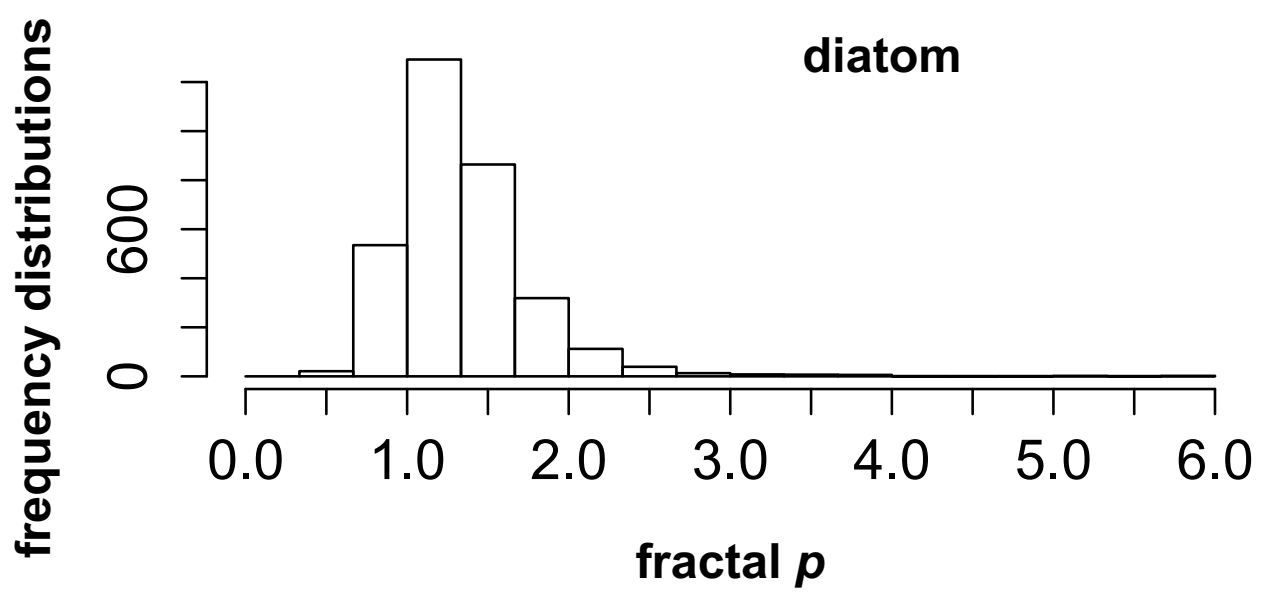

C

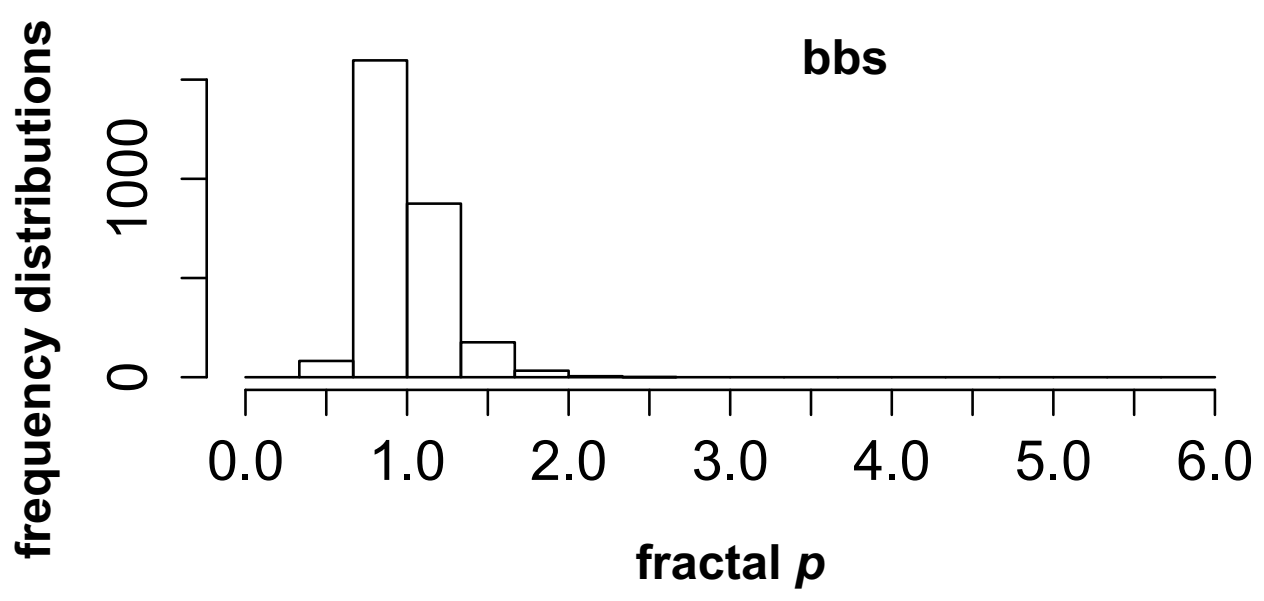

E

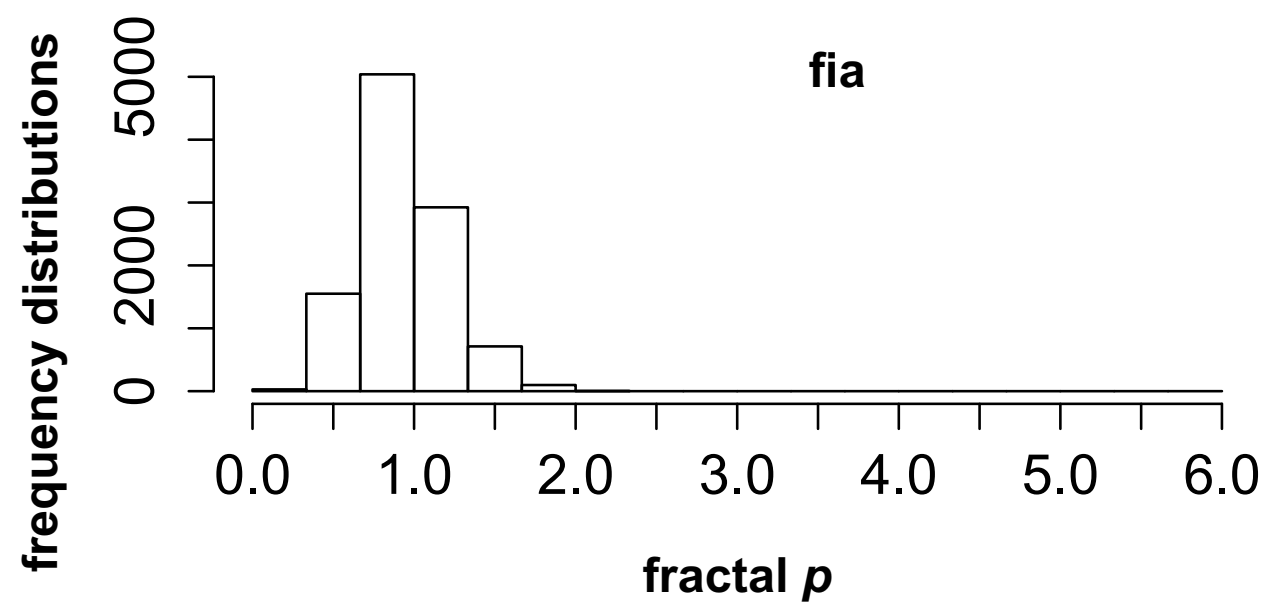

G

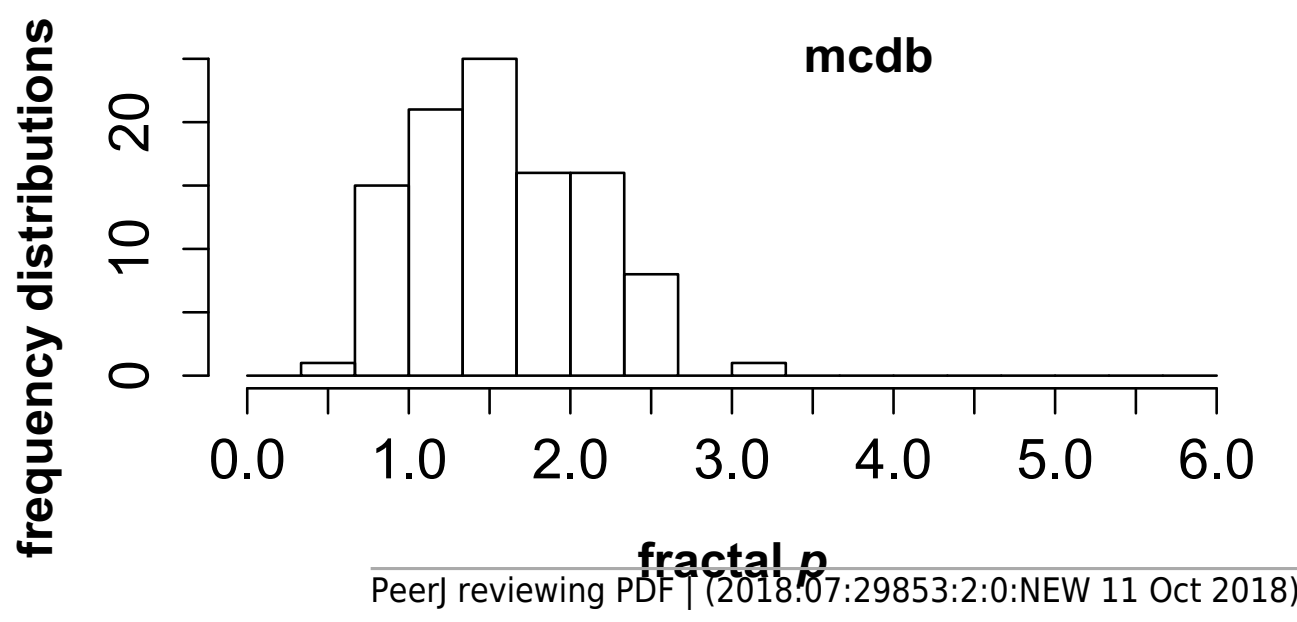

B Manuscript to be reviewed

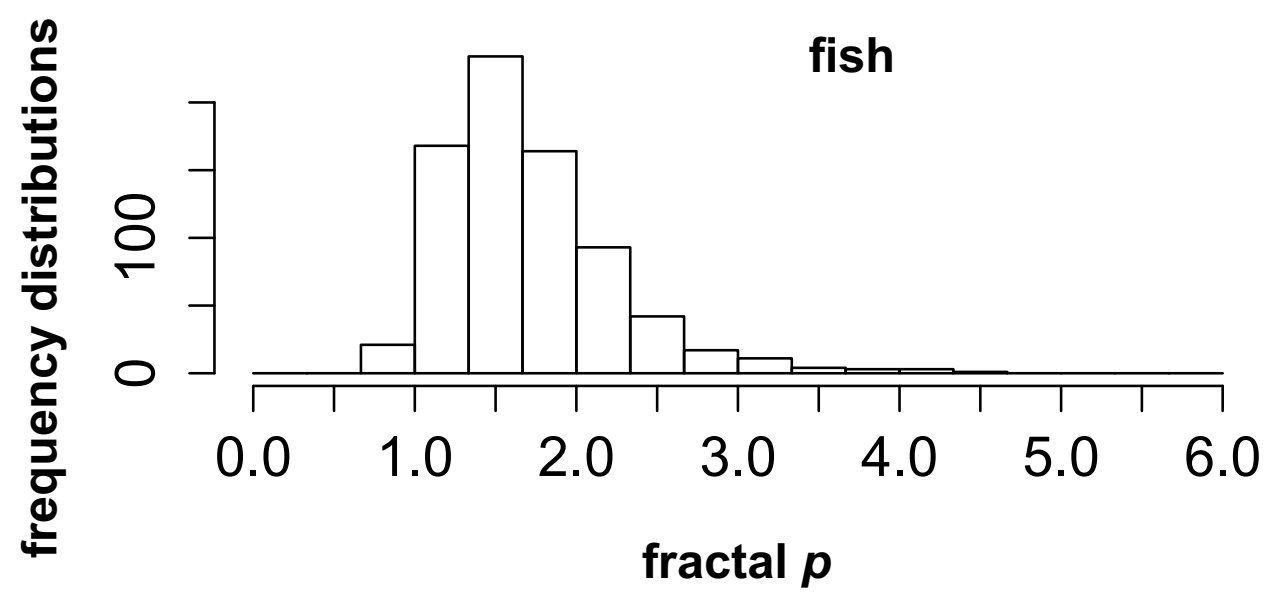

D

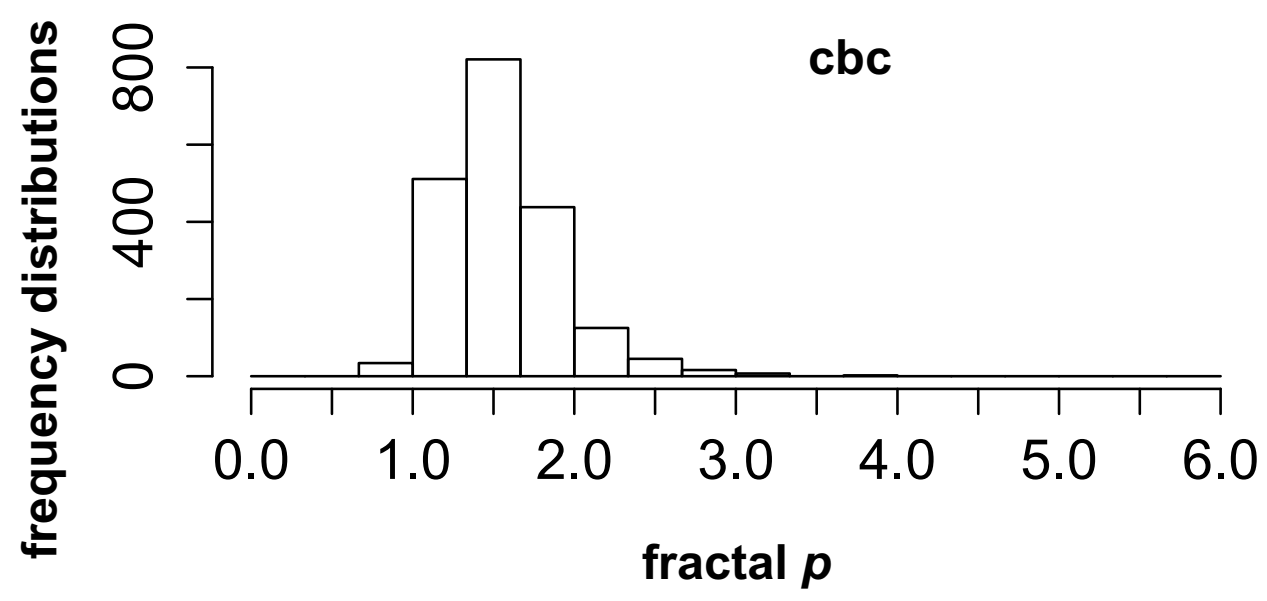

F

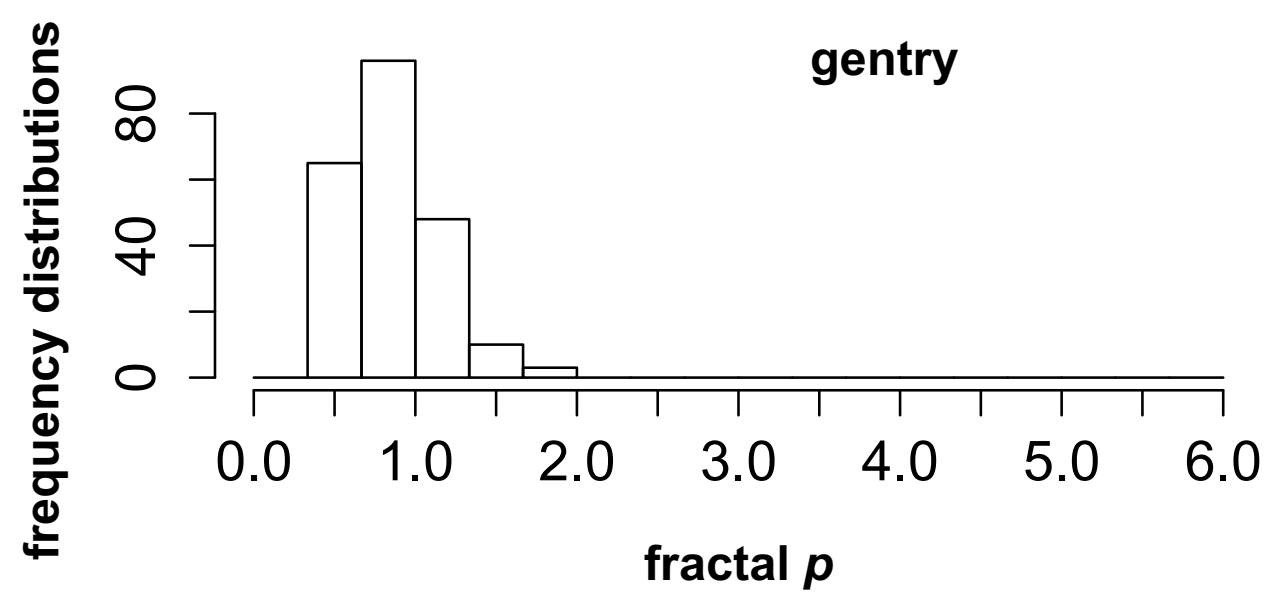

H

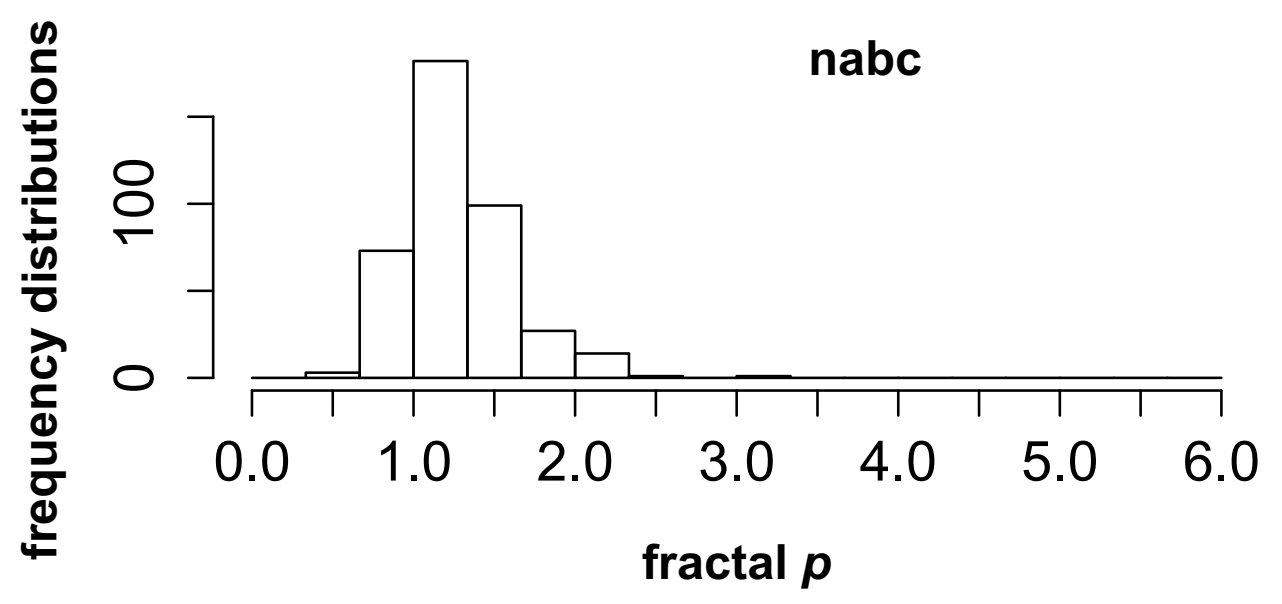

